CREAT. MATH. INFORM.

Volume 29 (2020), No. 2,

Pages 183 - 189

\title{
Further study on solutions of some non-linear homogeneous differential equations in connection to Brück conjecture
}

\author{
DILIP CHANDRA PRAMANIK and KAPIL ROY
}

\begin{abstract}
In this paper, using the theory of complex differential equations, we study the solution of some non-linear complex differential equations in connection to Brück conjecture which generalized some earlier results due to Pramanik, D. C. and Biswas, M., On solutions of some non-linear differential equations in connection to Bruck conjecture, Tamkang J. Math., 48 (2017), No. 4, 365-375; and Wang, H., Yang, L-Z. and Xu, H-Y., On some complex differential and difference equations concerning sharing function, Adv. Diff. Equ., 2014, 2014:274.
\end{abstract}

\section{INTRODUCTION}

In this paper, by meromorphic function we shall always mean a meromorphic function in the complex plane. We adopt the standard notations in the Nevanlinna Theory of meromorphic functions as explained in $[4,6,12]$. It will be convenient to let $E$ denote any set of positive real numbers of finite linear measure, not necessarily the same at each occurrence.

For any non-constant meromorphic function $f(z)$, we denote by $S(r, f)$ any quantity satisfying $S(r, f)=\circ(T(r, f))$ as $r \rightarrow \infty, r \notin E$. A meromorphic function $a(z)$ is said to be small with respect to $f(z)$ if $T(r, a)=S(r, f)$. We denote by $S(f)$ the collection of all small functions with respect to $f$. Clearly $\mathbb{C} \cup\{\infty\} \subset S(f)$ and $S(f)$ is a field over the set of complex numbers.

For any two non-constant meromorphic functions $f$ and $g$, and $a \in S(f) \cap S(g)$, we say that $f$ and $g$ share $a \mathrm{IM}(\mathrm{CM})$ provided that $f-a$ and $g-a$ have the same zeros ignoring(counting) multiplicities.

For $a \in \mathbb{C} \cup\{\infty\}$ the quantity defined by

$$
\delta(a, f)=\liminf _{r \rightarrow+\infty} \frac{m\left(r, \frac{1}{f-a}\right)}{T(r, f)}=1-\limsup _{r \rightarrow+\infty} \frac{N\left(r, \frac{1}{f-a}\right)}{T(r, f)}
$$

is called the deficiency of $a$ with respect to the function $f$.

Let $f(z)$ be a non-constant entire function, then the order $\sigma(f)$ of $f(z)$ is defined by

$$
\sigma(f)=\limsup _{r \rightarrow+\infty} \frac{\log T(r, f)}{\log r}=\limsup _{r \rightarrow+\infty} \frac{\log \log M(r, f)}{\log r}
$$

and the lower order $\mu(f)$ of $f(z)$ is defined by

$$
\mu(f)=\liminf _{r \rightarrow+\infty} \frac{\log T(r, f)}{\log r}=\liminf _{r \rightarrow+\infty} \frac{\log \log M(r, f)}{\log r}
$$

Received: 19.07.2019. In revised form: 08.09.2020. Accepted: 15.09.2020

2010 Mathematics Subject Classification. 39B32, 30D35.

Key words and phrases. entire function, Brück conjecture, small function, differential polynomial.

Corresponding author: Dilip Chandra Pramanik; dcpramanik.nbu2012@gmail.com 
where and in the sequel

$$
M(r, f)=\max _{|z|=r}|f(z)| .
$$

Let $n_{0 j}, n_{1 j}, n_{2 j}, \ldots, n_{k j}$ be non-negative integers. The expression

$$
M_{j}[f]=f^{n_{0 j}}\left(f^{(1)}\right)^{n_{1 j}}\left(f^{(2)}\right)^{n_{2 j}} \ldots\left(f^{(k)}\right)^{n_{k j}}
$$

is called a differential monomial generated by $f$ of degree $d\left(M_{j}\right)=\sum_{i=0}^{k} n_{i j}$ and weight $\Gamma_{M_{j}}=\sum_{i=0}^{k}(i+1) n_{i j}$. The sum

$$
P[f]=\sum_{j=1}^{l} a_{j} M_{j}[f],
$$

is called a differential polynomial generated by $f$ of degree $\bar{d}(P)=\max \left\{d\left(M_{j}\right): 1 \leq j \leq l\right\}$ and weight $\Gamma_{P}=\max \left\{\Gamma_{M_{j}}: 1 \leq j \leq l\right\}$, where $a_{j} \not \equiv 0$ and $T\left(r, a_{j}\right)=S(r, f)$ for $j=$ $1,2, \ldots, l$. The numbers $\underline{d}_{P}=\min \left\{d\left(M_{j}\right): 1 \leq j \leq l\right\}$ and $k$ (the highest order of the derivative of $f$ in $P[f]$ ) are called respectively the lower degree and the order of $P[f] . P[f]$ is said to be homogeneous differential polynomial of degree $d$ if $\bar{d}_{P}=\underline{d}_{P}=d$. $P[f]$ is called a linear differential polynomial if $\bar{d}_{P}=1$. Otherwise, $P[f]$ is called non-linear differential polynomial.

Let $f$ be a non-constant meromorphic function, then the hyper-order $\sigma_{2}(f)$ of $f(z)$ is defined as follows:

$$
\sigma_{2}(f)=\limsup _{r \rightarrow+\infty} \frac{\log \log T(r, f)}{\log r} .
$$

Rubel and Yang [10] proved that if a non-constant entire function $f$ and its derivative $f^{\prime}$ share two distinct finite complex numbers $\mathrm{CM}$, then $f \equiv f^{\prime}$. What will be the relation between $f$ and $f^{\prime}$, if an entire function $f$ and its derivative $f^{\prime}$ share one finite complex number $\mathrm{CM}$ ?

In 1996 Brück [1] made the following conjecture:

Conjecture 1.1. Let $f$ be a non-constant entire function satisfying $\sigma_{2}(f)<\infty$, where $\sigma_{2}(f)$ is not a positive integer. If $f$ and $f^{\prime}$ share one finite complex number a $C M$, then

$$
\frac{f^{\prime}-a}{f-a}=c,
$$

for some finite complex number $c \neq 0$.

Gundersen and Yang [3] proved that the conjecture is true for functions of finite order.

Theorem 1.1. Let $f$ be a non-constant entire function of finite order. If $f$ and $f^{\prime}$ share one finite complex number a CM, then

for some finite complex number $c \neq 0$

$$
\frac{f^{\prime}-a}{f-a}=c
$$

In 2009, Chang and Zhu [2] proved that Theorem 1.1 remains valid when the complex number $a$ is replaced by a small function of $f$.

Theorem 1.2. Let $f$ be a non-constant entire function of finite order and $a(z)$ be a function such that $\sigma(a)<\sigma(f)<\infty$. If $f$ and $f^{\prime}$ share $a(z) C M$, then

$$
\frac{f^{\prime}-a}{f-a}=c,
$$


for some finite complex number $c \neq 0$

In 2014, Wang et al [11] investigated the Brück conjecture and proved that the conjecture remains true when $f^{\prime}$ is replaced by the linear differential polynomial of $f$, namely $L(f)=a_{k}(z) f^{(k)}+a_{k-1}(z) f^{(k-1)}+\ldots+a_{1}(z) f^{\prime}+a_{0}(z) f$, where $a_{k}(z)(\not \equiv 0), \ldots, a_{0}(z)$ are polynomials.

Theorem 1.3. Let $f(z)$ be a transcendental entire function with $\sigma_{2}(f)<\infty$, let $\sigma_{2}(f)$ be not an integer. If $f$ and $L(f)$ share a constant $a C M$ and $\delta(0, f)>0$, then

$$
\frac{L(f(z))-a}{f(z)-a}=c
$$

for some non-zero constant $c$.

In 2017, Pramanik et al [9] investigated the growth of the non-constant entire solutions of some non-linear complex differential equations in connection to Brück conjecture using the theory of complex differential equation and proved the following theorem:

Theorem 1.4. Let $f(z)$ and $\alpha(z)$ be two non-constant entire functions satisfying $\sigma(\alpha)<\mu(f)$ and $\phi(z)$ be a polynomial. If $f$ is a non-constant entire solution of the following differential equation

then $\sigma_{2}(f)=\operatorname{deg} \phi$.

$$
P[f]-\alpha(z)=\left(f^{\bar{d}_{P}}-\alpha(z)\right) e^{\phi(z)},
$$

Regarding Theorems 1.3 and 1.4, one can ask the following questions: (i)What would happen when $L(f)$ and the constant $a$ were replaced respectively by a non-linear differential polynomial of $f$ and a small function with respect to $f$ in Theorem 1.3?

(ii)What will happen if $\phi(z)$ is an entire function in Theorem 1.4 ?

Keeping in mind the above questions, in this paper using the theory of complex differential equation we prove the following theorem:

Theorem 1.5. Let $f(z)$ be a transcendental entire function. Suppose that $\sigma_{2}(f)$ is not a positive integer or infinite and $\delta(0, f)>0$. Let $P[f]$ be a homogeneous differential polynomial of $f$ of degree $d$ and $\phi(z)$ be an entire function, and $\alpha(z) \in S(f)$ be such that $\sigma(\alpha)<\mu(f)$. If $f$ is a transcendental entire solution of the following differential equation

$$
P[f]-\alpha(z)=\left(f^{d}-\alpha(z)\right) e^{\phi(z)},
$$

then $\frac{P[f]-\alpha(z)}{f^{d}-\alpha(z)}=c$ for some non-zero constant $c$.

Remark 1.1. Following two examples support the main result Theorem 1.5 in the case when the differential polynomial $P[f]$ is homogeneous.

Example 1.1. Let $f(z)=e^{z}$ and $P[f]=f f^{\prime \prime}$. Clearly $\sigma_{2}(f)=0$ which is not a positive integer or infinity and $\delta(0 ; f)=1>0$ and $d=2$. Let $\alpha(z)$ be any polynomial in $z$. It is clear that $\alpha(z) \in S(f)$ and $\sigma(\alpha)=0<1=\mu(f)$, the transcendental entire function $f$ satisfies $P[f]-\alpha(z)=\left(f^{d}-\alpha(z)\right) e^{\phi(z)}$, where $\phi(z)=0$, an entire function, and we have $\frac{P[f]-\alpha(z)}{f^{d}-\alpha(z)}=c$, where $c=1$, a non-zero constant.

Example 1.2. Let $f(z)=e^{z}$ and $P[f]=a_{1} M_{1}[f]+a_{2} M_{2}[f]+a_{3} M_{3}[f]$, where $a_{1}, a_{2}$ and $a_{3}$ are constants be such that $a_{1}+a_{2}+a_{3}=1$, and $M_{1}[f]=f f^{2} f^{\prime}, M_{2}[f]=f^{2}\left(f^{\prime \prime}\right)^{2}$ and $M_{3}[f]=f^{3} f^{(k)}$. Clearly $\sigma_{2}(f)=0$ which is not a positive integer or infinity and $\delta(0 ; f)=1>0$ and $d=4$. Let $\alpha(z)$ be any polynomial in $z$. It is clear that $\alpha(z) \in S(f)$ and $\sigma(\alpha)=0<1=\mu(f)$, the transcendental entire function $f$ satisfies $P[f]-\alpha(z)=$ 
$\left(f^{d}-\alpha(z)\right) e^{\phi(z)}$, where $\phi(z)=0$, an entire function, and we have $\frac{P[f]-\alpha(z)}{f^{d}-\alpha(z)}=c$, where $c=1$, a non-zero constant.

Remark 1.2. The next example shows that in Theorem 1.5, one can not remove the condition $\sigma_{2}(f)$ is not a positive integer or infinity.

Example 1.3. Let $f(z)=e^{z}+c e^{e^{z}}$, where $c$ is any non-zero constant. Clearly, $\sigma_{2}(f)=1$ which is a positive integer and $\delta(0 ; f)>0$. Let $P[f]=f^{\prime}$ and $\alpha(z)=e^{z}$. Therefore, we see that $\alpha(z) \in S(f)$ and $\sigma(\alpha)=1<\infty=\mu(f)$, the transcendental entire function $f$ satisfies $P[f]-\alpha(z)=\left(f^{d}-\alpha(z)\right) e^{\phi(z)}$, where $\phi(z)=e^{z}$, an entire function, and we have $\frac{P[f]-\alpha(z)}{f^{d}-\alpha(z)}=e^{z} \neq c$, for any non-zero constant $c$.

\section{LEMMA}

In this section we state some lemmas needed in the sequel.

Lemma 2.1. [6] Let $f(z)$ be a transcendental entire function, $\nu(r, f)$ be the central index of $f(z)$. Then there exists a set $E \subset(1,+\infty)$ with finite logarithmic measure, we choose $z$ satisfying $|z|=r \notin[0,1] \cup E$ and $|f(z)|=M(r, f)$, we get

$$
\frac{f^{(j)}(z)}{f(z)}=\left\{\frac{\nu(r, f)}{z}\right\}^{j}(1+o(1)), \text { for } j \in N .
$$

Lemma 2.2. [5] Let $f(z)$ be an entire function of finite order $\sigma(f)=\sigma<+\infty$, and let $\nu(r, f)$ be the central index of $f$. Then

$$
\limsup _{r \rightarrow+\infty} \frac{\log \nu(r, f)}{\log r}=\sigma(f)
$$

And if $f$ is a transcendental entire function of hyper order $\sigma_{2}(f)$, then

$$
\limsup _{r \rightarrow+\infty} \frac{\log \log \nu(r, f)}{\log r}=\sigma_{2}(f)
$$

Lemma 2.3. [8] Let $f(z)$ be a transcendental entire function and let $E \subset[1,+\infty)$ be a set having finite logarithmic measure. Then there exists $\left\{z_{n}=r_{n} e^{i \theta_{n}}\right\}$ such that $\left|f\left(z_{n}\right)\right|=M\left(r_{n}, f\right), \theta_{n} \in$ $[0,2 \pi), \lim _{n \rightarrow+\infty} \theta_{n}=\theta_{0} \in[0,2 \pi), r_{n} \notin E$ and if $0<\sigma(f)<+\infty$, then for any given $\varepsilon>0$ and sufficiently large $r_{n}$,

$$
r_{n}^{\sigma(f)-\varepsilon}<\nu\left(r_{n}, f\right)<r_{n}^{\sigma(f)+\varepsilon} .
$$

If $\sigma(f)=+\infty$, then for any given large $K>0$ and sufficiently large $r_{n}$,

$$
\nu\left(r_{n}, f\right)>r_{n}^{K} \text {. }
$$

Lemma 2.4. [6] Let $P(z)=b_{n} z^{n}+b_{n-1} z^{n-1}+\ldots+b_{0}$ with $b_{n} \neq 0$ be a polynomial. Then for every $\varepsilon>0$, there exists $r_{0}>0$ such that for all $r=|z|>r_{0}$ the inequalities

$$
(1-\varepsilon)\left|b_{n}\right| r^{n} \leq|P(z)| \leq(1+\varepsilon)\left|b_{n}\right| r^{n}
$$

hold.

Lemma 2.5. [11] Let $f(z)$ and $A(z)$ be two entire functions with $0<\sigma(f)=\sigma(A)=\sigma<$ $+\infty, 0<\tau(A)=\tau(f)<+\infty$, then there exists a set $E \subset[1,+\infty)$ that has infinite logarithmic measure such that for all $r \in E$ and a positive number $\kappa>0$, we have

$$
\frac{M(r, A)}{M(r, f)}<\exp \left\{-\kappa r^{\sigma}\right\} .
$$


Lemma 2.6. [11] Let $g:(0, \infty) \rightarrow \mathbb{R}, h:(0, \infty) \rightarrow \mathbb{R}$ be monotone increasing functions such that $g(r) \leq h(r)$ outside of an exceptional set $E$ with finite linear measure, or $g(r) \leq h(r)$, $r \notin H \cup(0,1]$, where $H \subset(1, \infty)$ is a set of finite logarithmic measure. Then for any $\alpha>1$, there exists $r_{0}$ such that $g(r) \leq h(\alpha r)$ for all $r \geq r_{0}$.

\section{MAIN RESULTS}

In this section we present the proof of the main result of the paper.

Proof. We will consider the following two cases:

Case I: Let $\alpha(z) \equiv 0$. Then from (1.1) it follows that

$$
\frac{P[f]}{f^{d}}=e^{\phi(z)}
$$

Now,

$$
\begin{aligned}
\frac{M_{j}[f]}{f^{d}} & =\frac{f^{n_{0 j}}\left(f^{(1)}\right)^{n_{1 j}} \ldots\left(f^{(k)}\right)^{n_{k j}}}{f^{n_{0 j}+n_{1 j}+\ldots+n_{k j}}} \\
& =\left(\frac{f^{(1)}}{f}\right)^{n_{1 j}}\left(\frac{f^{(2)}}{f}\right)^{n_{2 j}} \ldots\left(\frac{f^{(k)}}{f}\right)^{n_{k j}}
\end{aligned}
$$

and so

$$
\begin{aligned}
m\left(r, \frac{M_{j}[f]}{f^{d}}\right) & =\sum_{i=1}^{k} n_{i j} m\left(r, \frac{f^{(i)}}{f}\right) \\
& =O(\log r T(r, f)), \text { for } j=1,2, \ldots, l .
\end{aligned}
$$

outside an exceptional set $E_{0}$ of finite linear measure.

From (3.2), it follows that

$$
\begin{aligned}
T\left(r, e^{\phi(z)}\right) & =m\left(r, e^{\phi(z)}\right)=m\left(r, \frac{P[f]}{f^{d}}\right) \\
& \leq \sum_{j=1}^{l} m\left(r, \frac{M_{j}[f]}{f^{d}}\right)+\sum_{j=1}^{l} m\left(r, a_{j}\right)+\log k \\
& =O(\log r T(r, f)),
\end{aligned}
$$

outside an exceptional set $E_{0}$ of finite linear measure.

Thus there exists a constant $K$ such that

$$
T\left(r, e^{\phi(z)}\right) \leq K \log (r T(r, f)) \text { for } r \notin E_{0} .
$$

By Lemma 2.6 there exists an $r_{0}>0$ such that for all $r \geq r_{0}$, we have

$$
T\left(r, e^{\phi(z)}\right) \leq K \log (\eta r T(\eta r, f)) \text { for } \eta>1 .
$$

From (3.3), we can deduce that $\sigma\left(e^{\phi(z)}\right) \leq \sigma_{2}(f)<\infty$ and hence $\phi(z)$ is a polynomial.

Proceeding similarly as in [Theorem 3, [9]], we obtain that $\sigma_{2}(f)=\operatorname{deg} \phi$, which is a contradiction to our assumption that $\sigma_{2}(f)$ is not an integer. Hence $\phi(z)$ is only a constant.

Case II: Let $\alpha(z) \not \equiv 0$. Taking the logarithmic derivative of (1.1), we get that

$$
\phi^{\prime}(z)=\frac{P^{\prime}[f]-\alpha^{\prime}(z)}{P[f]-\alpha(z)}-\frac{d f^{d-1} f^{\prime}-\alpha^{\prime}(z)}{f^{d}-\alpha(z)} .
$$

Subcase I: Let $\phi^{\prime}(z) \equiv 0$. Then $\phi(z)=c_{1}, c_{1}$ is a constant. 
Subcase II: Let $\phi^{\prime}(z) \not \equiv 0$. Then it follows from (3.4) that

$$
m\left(r, \phi^{\prime}\right)=S(r, f) \text {. }
$$

We can rewrite (3.4) in the following form:

$$
\begin{aligned}
\phi^{\prime} & =f^{d}\left[\frac{P[f]}{f^{d}} \cdot \frac{1}{P[f]} \cdot \frac{P^{\prime}[f]-\alpha^{\prime}(z)}{P[f]-\alpha(z)}-\frac{1}{f^{d}} \frac{d f^{d-1} f^{\prime}-\alpha^{\prime}(z)}{f^{d}-\alpha(z)}\right] \\
& =\frac{f^{d}}{\alpha(z)}\left[\frac{P[f]}{f^{d}} \cdot \frac{P^{\prime}[f]-\alpha^{\prime}(z)}{P[f]-\alpha(z)}-\frac{P^{\prime}[f]}{f^{d}}-\frac{d f^{d-1} f^{(1)}-\alpha^{\prime}(z)}{f^{d}-\alpha(z)}+\frac{d f^{\prime}}{f}\right] .
\end{aligned}
$$

Since $\phi \not \equiv 0$ and $f$ is a transcendental entire function, we set

$$
\psi=\frac{P[f]}{f^{d}} \cdot \frac{P^{\prime}[f]-\alpha^{\prime}(z)}{P[f]-\alpha(z)}-\frac{P^{\prime}[f]}{f^{d}}-\frac{d f^{d-1} f^{(1)}-\alpha^{\prime}(z)}{f^{d}-\alpha(z)}+\frac{d f^{\prime}}{f} .
$$

Then we have

$$
m(r, \psi)=S(r, f) .
$$

Therefore it follows from (3.6) and (3.7) that

$$
\frac{\alpha(z)}{f^{d}}=\frac{\psi(z)}{\phi^{\prime}(z)} .
$$

Since $\phi$ is an entire function, then we have

$$
\begin{aligned}
m\left(r, \frac{1}{f^{d}}\right) & \leq m\left(r, \frac{\alpha(z)}{f^{d}}\right)+m\left(r, \frac{1}{\alpha(z)}\right) \\
& \leq m\left(r, \frac{\psi(z)}{\phi^{\prime}(z)}\right)+S(r, f) \\
& \leq m(r, \psi(z))+m\left(r, \frac{1}{\phi^{\prime}(z)}\right)+S(r, f) \\
& \leq T\left(r, \phi^{\prime}(z)\right)+S(r, f) \\
& =m\left(r, \phi^{\prime}\right)+S(r, f) \\
& =S(r, f) \\
\Rightarrow m\left(r, \frac{1}{f}\right) & =S(r, f) .
\end{aligned}
$$

It follows from (3.9) that

$$
\delta(0, f)=\liminf _{r \rightarrow \infty} \frac{m\left(r, \frac{1}{f}\right)}{T(r, f)}=0,
$$

which is a contradiction.

Thus the proof is completed.

Conclusion: The paper is intended to study the growth of solutions of complex differential equations which is of great importance of its own. Many researchers have worked on this particular field and high up the level of it to a new dimension. We think that our work will contribute a little to the existing literature and help the future researchers of this and related fields. We have investigated those solutions of (1.1) which have deficiency at the origin. Therefore we are posing the following question for further investigation:

Question: What can be said about the solution of (1.1) if $\mathrm{f}$ has no deficient value? Acknowledgment. Authors would like to thank referees for their valuable comments and suggestions. 


\section{REFERENCES}

[1] Brück, R., On entire functions which share one value CM with their first derivative, Results Math., 30 (1996), 21-24

[2] Chang, J. M. and Zhu, Y. Z., Entire functions that share a small function with their derivatives, J. Math. Anal. Appl., 351 (2009), 491-496

[3] Gundersen, G. G. and Yang, L. Z., Entire functions that share one value with one or two of their derivatives, J. Math. Anal. Appl., 223 (1998), 85-95

[4] Hayman, W. K., Meromorphic function, Clarendon Press, Oxford, 1964

[5] He, Y. Z. and Xiao, X. Z., Algebroid functions and ordinary differential Equations, Science press, Beijing, 1998

[6] Laine, I., Nevanlinna Theory and Complex Differential Equations, Walter de Gruyter, Berlin, 1993

[7] Li, X. M. and Cao, C. C., Entire functions sharing one polynomial with their derivatives, Proc. Indian Acad. Sci. Math. Sci., 118 (2008), 13-26

[8] Mao, Z. Q., Uniqueness theorems on entire functions and their linear differential polynomials, Results Math., 55 (2009), 447-456

[9] Pramanik, D. C. and Biswas, M., On solutions of some non-linear differential equations in connection to Brück conjecture, Tamkang J. Math., 48 (2017), No. 4, 365-375

[10] Rubel, L. A. and Yang, C. C., Values shared by an entire function and its derivative, Lecture Notes in Math., Springer-Verlag, Berlin, 599 (1977), 101-103

[11] Wang, H., Yang, L-Z. and Xu, H-Y., On some complex differential and difference equations concerning sharing function, Adv. Diff. Equ., 2014, 2014:274

[12] Yang, L., Value distributions theory, Springer-Verlag, Berlin, 1993

DEPARTMENT OF MATHEMATICS

UNIVERSITY OF NORTH BENGAL

RAJA RAMMOHUNPUR, DARJEELING-734013, WEST BENGAL, INDIA

Email address: dcpramanik.nbu2012@gmail.com, roykapil692@gmail.com 